et posons $x=\xi_{1}+x_{m, \varepsilon}, y=\eta_{1}+x_{m, \varepsilon}$ (où $x_{m, \varepsilon}$ est la fonction du Lemme 2). On a $\left|x_{m, \varepsilon}(t)\right| \leqslant \frac{1}{2} \varepsilon$, donc $\varrho(\xi, x)<\varepsilon, \varrho(\eta, y)<\varepsilon$. Il suffit donc de démontrer que $[x, y] \epsilon G_{n}$.

Posons $x y=z, \quad \xi_{1} \eta_{1}=z_{1}, \quad \xi_{1} x_{m, \varepsilon}=z_{2}, \quad \eta_{1} x_{m, \varepsilon}=z_{3}, \quad x_{m, \varepsilon} x_{m, 8}=z_{4}$ donc $z=z_{1}+z_{2}+z_{3}+z_{4}$. Si $0 \leqslant t \leqslant 1, \quad 0 \leqslant u \leqslant 1, t \neq u$, on a, d'après le Lemme 1 ,

$$
\left|\sum_{i=1}^{3} \frac{z_{i}(t)-z_{i}(u)}{t-u}\right| \leqslant 2 A \cdot A+2 A \cdot \frac{1}{2} \varepsilon+2 A \cdot \frac{1}{2} \varepsilon<4 A^{2} .
$$

Soit maintenant $1 / n \leqslant t \leqslant 1-1 / n$. Choisissons $r, s$ comme il suit. $\Pi$ existe un nombre entier $k$ tel que

$$
\frac{2 k+1}{2 m} \leqslant t<\frac{2 k+3}{2 m} ;
$$

on a $k>0$, car $3 / 2 m<1 / n \leqslant t$; posons

$$
r=\frac{2 k+1}{2 m}, \quad s=\frac{2 k+6}{2 m},
$$

donc $0<\operatorname{Max}(s-t, t-r) \leqslant s-r=5 / 2 m<1 / n$, d'où $0<r \leqslant t<s<1$. Le Lemme 2 donne

$$
\frac{z_{4}(s)-z_{4}(r)}{s-r}=\varepsilon^{2}\left(\frac{2 k+6}{24 m}+\frac{2 k+1}{24 m}\right) \cdot \frac{2 m}{5}>\varepsilon^{2} \cdot \frac{t}{12} \cdot \frac{2 m}{5} \geqslant \frac{m \varepsilon^{2}}{30 n} .
$$

Or, (24), (25), (23) donnent

$$
\frac{z(s)-z(r)}{s-r}>\frac{m \varepsilon^{2}}{30 n}-4 A^{2}>n,
$$

ce qui démontre que $[x, y] \epsilon G_{n}$.

(Reşu par la Rédaction le 2. 5. 1950).

\section{On the ergodic theorems (I)}

(Generalized ergodic theorems)

C. RYLL-NARDZEWSKI (Wrocław).

\section{Introduction.}

In this paper ${ }^{1}$ ) we understand by space a fixed abstract set $X$ with a $\sigma$-finite $\sigma$-measure $\mu$ defined in a $\sigma$-field $M$ of subsets of $X$. By sets we always understand sets belonging to $M$. The letter $\varphi$ will be used for a transformation of $X$ into itself and we shall assume $\varphi^{-1} E \in M$ for $E \in M$ and $\mu\left(\varphi^{-1} E\right)=0$ if $\mu(E)=0$. By functions we understand only the real-valued functions defined on $X$, and measurable with respect to $M$. The letters $f$ and $g$, with indices if necessary, will always denote functions. The class of all $f$ integrable with respect to $\mu$ will be denoted by $L(\mu)$. The symbol $\int$ always denotes the integral over the whole space. The symbol $[\mu]$ placed after an equality or an inequality means that it is fulfilled almost everywhere (with respect to $\mu$ ).

The individual and mean ergodic theorems of BIRKноFF and v. NEUMANN (generalized by F. RLesz [6]) state that if $\varphi$ is measurepreserving (i.e. if $\left.\mu\left(\varphi^{-1} E\right)=\mu(E)\right)$, then

(B) for each $f \in L(\mu)$ there is a $g \in L(\mu)$ such that

$$
\lim _{n} \frac{1}{n} \sum_{i=0}^{n-1} f\left(\varphi^{i}(x)\right)=g(x)[\mu],
$$

and (if $\mu(X)<\infty$ )

(N) for each $f \in L(\mu)$ there is a $g \in L(\mu)$ such that

$$
\lim _{n} \int\left|\frac{1}{n} \sum_{i=0}^{n-1} f\left(\varphi^{i}(x)\right)-g(x)\right| d \mu=0 .
$$

1) Presented to the Polish Mathematical Society, Wroclaw Section, on March 10, 1950. Cf. [5].

Studia Mathematica. T. XII 
DUNFORD and MILLER [4] have formulated the following condition:

(DM) There is a constant $K$ such that

$$
\frac{1}{n} \sum_{i=0}^{n-1} \mu\left(\varphi^{-i} E\right) \leqslant K \mu(E)
$$

for each set $E$ and $n=1,2, \ldots$

DUNFORD and MILLER proved that under the assumption $\mu(X)<\infty$ in the preceding ergodic theorems the preservation of measure by $\varphi$ may be replaced by (DM). More exactly: the statements (DM) and (N) are equivalent and imply (B).

S. HARTMAN recently formulated the following condition:

(H) There is a constant $K$ such that

$$
\varlimsup_{n} \frac{1}{n} \sum_{i=0}^{n-1} \mu\left(\varphi^{-i} E\right) \leqslant K \mu(E)
$$

for each set $E$.

Obviously (DM) implies (H).

The main result of this paper (Theorem 1) may be formulated for finite measure as follows: (H) and (B) are equivalent. For $\sigma$-finite measures the condition (H) must be replaced by related conditions $\left(\mathrm{H}_{1}\right),\left(\mathrm{H}_{2}\right)$ or $\left(\mathrm{H}_{3}\right)$.

We shall also prove the result of DUNFORD and MIrLER (Theorem 2). The implication $(\mathrm{DM}) \rightarrow(\mathrm{N})$ will be proved by means of Theorem 1 and the converse implication by a part of the original Dunford-Miller's proof.

Moreover we prove by a counter-example that $(\mathbf{H})$ does not imply (DM), and that consequently (B) does not imply (N). Our example is a modification of those of Y.N. DowkER [3].

In the proof of Theorem 1 we use the individual ergodic theorem fomulated above and the construction of the auxiliary invariant measure made in a paper by DOWKER [2].

\section{Generalized individual ergodic theorem.}

Given a transformation $\varphi$, put for each two sets $A$ and $Y$

$$
M_{n}(A, Y)=\frac{1}{n} \sum_{k=0}^{n-1} \mu\left(Y \cdot \varphi^{-k} A\right)
$$

and consider three conditions (where $K$ is a constant number):

(i) for each $A$ and each $Y$ with $\mu(Y)<\infty$ we have

$$
\lim _{n} M_{n}(A, Y) \leqslant K \mu(A) \text {; }
$$

(ii) for each $A$ and each $Y$ with $\mu(Y)<\infty$ we have

$$
\varlimsup_{n} M_{n}(A, Y) \leqslant K \mu(A) ;
$$

(iii) there is an ascending sequence $\left\{Y_{j}\right\}$ of sets such that $X=Y_{1}+Y_{2}+\ldots$ and (2) holds for $Y=Y_{j} \quad(j=1,2, \ldots)$ and each set $A$.

We say that $\varphi$ possesses the property $\left(\mathrm{H}_{1}\right),\left(\mathrm{H}_{2}\right)$ or $\left(\mathrm{H}_{3}\right)$ if there is a constant $K$ such that (i), (ii) or (iii) holds respectively.

Obviously in the case $\mu(X)<\infty$ the condition $\left(\mathrm{H}_{2}\right)$ is equivalent to the condition (H) formulated above in the introduction.

Theorem 1. The statement (B) is equivalent to each of the statements $\left(\mathrm{H}_{j}\right)(j=1,2,3)$.

Since obviously $\left(\mathrm{H}_{1}\right)$ implies $\left(\mathrm{H}_{2}\right)$ and $\left(\mathrm{H}_{2}\right)$ implies $\left(\mathrm{H}_{3}\right)$, it suffices to prove: $1^{0}\left(\mathrm{H}_{3}\right)$ implies (B); $2^{0}(\mathrm{~B})$ implies $\left(\mathrm{H}_{1}\right)$.

We may suppose that the sequence $\left\{Y_{j}\right\}$ in $\left(\mathrm{H}_{3}\right)$ satisfies the condition $\mu\left(Y_{j}\right)<\infty$, since otherwise we may replace the sets $Y_{j}$ by $X_{j} Y_{j}$, where $\left\{Y_{j}\right\}$ is an ascending sequence of sets of finite measure the sum of which is $X$.

$1^{0}$ Let Lim $u_{n}$ be the Mazur-Banach generalized limit ${ }^{2}$ ), i.e. a functional defined for all bounded sequences of real numbers which satisfies the following conditions:

$$
\begin{aligned}
& \text { I. } \operatorname{Lim}_{n}\left(a u_{n}+b v_{n}\right)=a \operatorname{Lim}_{n} u_{n}+b \operatorname{Lim}_{n} v_{n} ; \\
& \text { II. } \quad \operatorname{Lim}_{n+1} u_{n+1} \operatorname{Lim}_{n} ; \\
& \text { III. } \quad \frac{\lim u_{n} \leqslant \operatorname{Lim}_{n} u_{n} \leqslant \varlimsup_{n} u_{n} .}{}
\end{aligned}
$$

We define a set function $\nu_{j}(A)$ putting

$$
v_{j}(A)=\operatorname{Lim}_{n} M_{n}\left(A, Y_{j}\right),
$$

\footnotetext{
2) Banach [1], p. 34 .
} 
which is possible, since the sequence $M_{n}\left(A, X_{j}\right)$ is bounded on account of $\left(\mathrm{H}_{3}\right)$. The function $v_{j}$ has the following properties:

$$
\begin{array}{ll}
\left(\alpha_{j}\right) \quad 0 \leqslant v_{j}(A) \leqslant K \mu(A) ; \\
\left(\beta_{j}\right) \quad v_{j}(A+B)=v_{j}(A)+v_{j}(B) \quad \text { if } A B=0 ; \\
\left(\gamma_{j}\right) \quad \text { if } A=\varphi^{-1} A, \text { then } v_{j}(A)=\mu\left(A Y_{j}\right) ; \\
\left(\delta_{j}\right) \quad v_{j}\left(\varphi^{-1} A\right)=v_{j}(A) .
\end{array}
$$

Property $\left(\alpha_{j}\right)$ follows directly from $\left(\mathrm{H}_{3}\right)$ and III. Property $\left(\beta_{j}\right)$ follows directly from $\mathrm{I}$.

If $A=\varphi^{-1} A$, then $M_{n}\left(A, Y_{j}\right)=\mu\left(A Y_{j}\right)$, whence, in virtue of III, $v_{j}(A)=\mu\left(A Y_{j}\right)$. Thus we obtain the property $\left(\gamma_{j}\right)$.

On account of $I$ we have

$$
\nu_{j}(A)-\nu_{j}\left(\varphi^{-1} A\right)=\underset{n}{\operatorname{Lim}}\left[M_{n}\left(A, Y_{j}\right)-M_{n}\left(\varphi^{-1} A, Y_{j}\right)\right]
$$

and from the definition of $M_{n}$ we get

$$
\left|M_{n}\left(A, Y_{j}\right)-M_{n}\left(\varphi^{-1} A, Y_{j}\right)\right| \leqslant \frac{2}{n} \mu\left(Y_{j}\right)
$$

Hence, in virtue of III, we obtain $\left(\delta_{j}\right)$.

The sequence $\left\{v_{j}(A)\right\}$ being non-decreasing for fixed $A$ we may put

$$
v(A)=\lim _{j} v_{j}(A)
$$

(where $v(A)$ is finite or not).

The properties $\left(\alpha_{j}\right)-\left(\delta_{j}\right)$ imply directly the following properties of $\nu$ :

$$
\begin{array}{ll}
(\alpha) & 0 \leqslant v(A) \leqslant K \mu(A) ; \\
(\beta) \quad & v(A+B)=v(A)+v(B) \quad \text { if } \quad A B=0 ; \\
(\gamma) \quad \text { if } A=\varphi^{-1} A, \text { then } \quad v(A)=\mu(A) ; \\
(\delta) \quad v\left(\varphi^{-1} A\right)=v(A) .
\end{array}
$$

It follows from $(\alpha)$ and $(\beta)$ that $\nu$ is a $\sigma$-finite $\sigma$-measure.

Since the measure $\nu$ is invariant with respect to $\varphi$ (property $(\delta)$ ), we may apply the individual ergodic theorem (see Introduction). Thus $\varphi$ satisfies the condition (B) with respect to the measure $v$. Now we shall prove that (B) is satisfied with respect to $\mu$.
Let $f \in L(\mu)$. It follows from $(a)$ that $f \in L(\nu)$. Therefore, there is a function $g \in L(v)$ such that

(3) $\quad \lim _{n} \frac{1}{n}\left[f(x)+f(q(x))+\ldots+f\left(\varphi^{n-1}(x)\right)\right]=g(x) \quad$ for $\quad x \bar{\epsilon} D$ where

and

$(4)$

$$
\nu(D)=0, \quad \varphi^{-1} D=D,
$$

( $D$ denotes the set of all divergence points of the sequence appearing in the formula (3)).

It follows from $(\gamma)$ that $\mu(D)=0$. In virtue of $(4)$. and $(\gamma)$, the Lebesgue sums which define the integrals of $g$ with respect to $\mu$ and $v$ are identical, whence $g \in L(\mu)$.

The implication $1^{0}$ is thus proved.

$2^{\circ}$ We first prove the following

Lemma 1. If $T$ is a mapping of $L(\mu)$ into itself which satisfies the following conditions:

$$
\begin{aligned}
& \text { (a) if } f=g[\mu] \text {, then } T f=T g[\mu] \text {; } \\
& \text { (b) } T(\alpha f+\beta g)=\alpha T f+\beta T g[\mu] \text { for real } \alpha \text { and } \beta \text {; } \\
& \text { (c) if } f \geqslant 0[\mu] \text {, then } T f \geqslant 0[\mu] \text {; }
\end{aligned}
$$

then $T$ is continuous, i.e. there is a constant $K$ such that

$$
\int|T f| d \mu \leqslant K \int|f| d \mu \quad \text { for } \quad f \in L(\mu) .
$$

It is sufficient to consider only $f \geqslant 0$. If the thesis does not hold, then there is a sequence $f_{n} \geqslant 0$ such that

$$
\int f_{n} d \mu=1, \quad \text { and } \quad \int\left(T f_{n}\right) d \mu>n^{2} .
$$

Therefore, there exists a function $f \in L(\mu)$ such that

$$
f=\sum_{n=1}^{\infty} \frac{1}{n^{2}} f_{n}[\mu] \text {. }
$$

Consequently, on account of (a)-(c), we have

$$
\int(T f) d \mu \geqslant \int T\left(\sum_{n=1}^{N} \frac{1}{n^{2}} f_{n}\right) d \mu=\sum_{n=1}^{N} \frac{1}{n^{2}} \int\left(T f_{n}\right) d \mu>N .
$$


Thus, the function $T f$ is non-integrable, which contradicts the hypothesis. The Lemma is thus proved.

Since the condition (B) is satisfied, we may consider for each $f \in L(\mu)$ a function $T f \epsilon L(\mu)$ such that

$$
T f=\lim _{n} \frac{1}{n} \sum_{i=0}^{n-1} f\left(\varphi^{i}(x)\right)[\mu] .
$$

Of course the mapping $T$ satisfies the conditions (a)-(c) of Lemma 1 (the condition (a) follows from the fact that $\mu\left(\varphi^{-1} E\right)=0$ if $\mu(E)=0$ ), and consequently there is a constant $K$ such that the formula (5) holds.

Let $Y$ be a set with $\mu(Y)<\infty$ and $f_{1}$ the characteristic function of a set $A$. Then the function $f_{k}(x)=f_{1}\left(\varphi^{k-1}(x)\right)$ is the characteristic function of the set $\varphi^{-k+1} A$; hence

$$
M_{n}(A, Y)=\int_{Y} \frac{1}{n}\left(f_{1}+f_{2}+\ldots+f_{n}\right) d \mu .
$$

Since, on account of $(5)$,

$$
\begin{gathered}
\lim _{n} \int_{Y} \frac{1}{n}\left(f_{1}+f_{2}+\ldots+f_{n}\right) d \mu=\int_{Y} \lim _{n} \frac{1}{n}\left(f_{1}+f_{2}+\ldots+f_{n}\right) d \mu \\
\leqslant \int T f_{1} d \mu \leqslant K \int f_{1} d \mu=K \mu(A),
\end{gathered}
$$

we obtain (i). In other words, the implication $(\mathrm{B}) \rightarrow\left(\mathrm{H}_{1}\right)$ is proved.

This proof continues to hold, if we replace the convergence almost everywhere in (B) by the convergence in measure.

\section{Generalized mean ergodic theorem.}

We suppose in this section $\mu(X)<\infty$.

Let $\Phi f$ denote the function $f(\varphi(x))$ (defined for all $x \in X$ ). We shall write $\Phi_{n} f$ for $\left(f+\Phi f+\ldots+\Phi^{n-1} f\right) / n$.

Obviously if $(\mathbb{N})$, then $\Phi f \epsilon L(\mu)$ for $f \epsilon L(\mu)$. Therefore, in virtue of Lemma 1, we get

Lemma 2. If $(\mathrm{N})$, then $\Phi$ is a continuous linear operator in the space $L(\mu)$.

Lemma 3. For the operator $\Phi$ to fulfil condition (5) (in which $T$ is replaced by $\Phi$ ) for each integrable $f$ it is sufficient that $\Phi$ fulfil (5) for the characteristic function $f$ of each set.
In fact, then $\Phi$ also fulfils (5) for each function assuming a finite number of values and consequently (by passing to the limit) for each $f \in L(\mu)$.

Lemma 4. The transformation $\varphi$ satisfies (DM) if and only if $\Phi$ is a continuous linear operator in $L(\mu)$ and the sequence of the operators $\Phi_{n}$ is bounded, i.e. there is a constant $M$ such that

$$
\int\left|\Phi_{n} f\right| d \mu \leqslant M \int|f| d \mu
$$

$n=1,2, \ldots$

for $f \in L(\mu)$.

The sufficiency is obvious: if $f$ in (6) is the characteristic function of a set $E$, we obtain (DM)

To prove the necessity, we use (DM) for $n=2$, whence

$$
\mu\left(\varphi^{-1}(E)\right) \leqslant(2 M-1) \mu(E),
$$

which shows in view of Lemma 3 that $\Phi$ is a continuous operator in $L(\mu)$. The condition (DM) states that the characteristic function $f$ of each set fulfils (6), whence each finitely-valued and consequently each integrable $f$ does the same.

Theorem 2. The statements (N) and (DM) are equivalent.

Proof. $1^{0}(\mathrm{DM}) \rightarrow(\mathrm{N})$. Obviously the statement $(\mathrm{DM})$ implies (H). From Theorem 1 we conclude that for each integrable $f$ the sequence $\Phi_{n} f$ converges almost everywhere to an integrable function. If $f$ is bounded, then $\Phi_{n} f$ is a uniformly bounded sequence of functions, and, since $\mu(X)<\infty$, this sequence converges in mean. The set of bounded functions is dense in the space $L(\mu)$. In view of Lemma 4, the sequence of operators $\Phi_{n}$ is bounded. Hence, on account of a BANACH-STEINHAUs theorem $\left.{ }^{3}\right) \Phi_{n} f$ converges in mean for each $f \in L(\mu)$.

$2^{0}(\mathrm{~N}) \rightarrow(\mathrm{DM})$. We see by Lemma 2 that $\Phi$ is a continuous linear operator in $L(\mu)$. The convergence in mean of $\Phi_{n} f$ for each $f \epsilon L(\mu)$ implies (by another BANACH-STEINHAUs theorem ${ }^{4}$ )) that the sequence of operators $\Phi_{n}$ is bounded. By Lemma 4, we obtain (DM).

\section{A counter-example.}

We shall prove by a counter-example that the statement $(\mathrm{H})$ does not imply the statement (DM). For this purpose we shall define

3) Banach [1], p. 79, Théorème 3 .

4) Banach [1], p. 80, Théorème 5 . 
a finite measure $\mu$ in the field of all subsets of a denumerable space $X$ and a one-one transformation $\varphi$ of $X$ on itself such that for $E \subset X$

$$
\begin{gathered}
\frac{1}{2} \mu(E) \leqslant \mu\left(\varphi^{-1} E\right) \leqslant 2 \mu(E), \\
\lim _{n} M_{n}(E, X) \leqslant 2 \mu(E)
\end{gathered}
$$

(where $M_{n}$ is defined by (1)) and

$$
\sup _{E} \sup _{n} \frac{M_{n}(E, X)}{\mu(E)}=\infty
$$

(where $E$ runs over the class of all sets of positive measure).

Let $X_{1}, X_{2}, \ldots$ be a sequence of finite disjoint sets, such that $X_{k}$ possesses $2^{k+1}$ points. For simplicity's sake the points of $X_{k}$ for a fixed $k$ shall be denoted by $1,2, \ldots, 2^{k+1}$.

We define a measure $\nu$ in $X$ and a transformation $\varphi$ by putting

$$
\nu(j)= \begin{cases}2^{j-1} & \text { for } \quad 1 \leqslant j \leqslant k, \\ 2^{2 k-j} & \text { for } \quad k<j \leqslant 2 k, \\ 1 & \text { for } \quad 2 k<j \leqslant 2^{k+1},\end{cases}
$$

and

$$
\varphi(j)=\left\{\begin{array}{lcc}
2^{k+1} & \text { for } & j=1, \\
j-1 & \text { for } & 1<j \leqslant 2^{k+1} .
\end{array}\right.
$$

The space $X_{k}$ and the measure $v$ satisfy (7), (8) and

$$
\sup _{E} \sup _{n} \frac{M_{n}\left(E, X_{k}\right)}{\nu(E)} \geqslant \frac{2^{k}-1}{k} \text {. }
$$

Formula (7) is obvious. In order to prove (8) it suftices to consider a one-point set $E=\left(j_{0}\right)$ and consequently, since $\varphi$ has a period $2^{k+1}$, it is enough to verify the inequality

$$
M_{2^{k+1}}\left(\left(j_{0}\right), X_{k}\right) \leqslant 2 v\left(\left(j_{0}\right)\right),
$$

which is proved as follows:

$$
\begin{aligned}
& M_{2^{k+1}}\left(\left(j_{0}\right), X_{k}\right)=\frac{1}{2^{k+1}} \sum_{i=0}^{2^{k+1}-1} \nu\left[\varphi^{-i}\left(j_{0}\right)\right] \\
& =\frac{\nu\left(X_{k}\right)}{2^{k+1}}=\frac{1}{2^{k+1}}\left(2^{k+2}-2-2 k\right) \leqslant 2 \nu\left(j_{0}\right) .
\end{aligned}
$$

It is easy to prove

$$
\frac{M_{k}\left((1), X_{k}\right)}{\nu(1)}=\frac{2^{k}-1}{k},
$$

which implies $\left(9^{\prime}\right)$.

We now define a measure $\mu$ in the whole space $X=X_{1}+X_{2}+\ldots$ by putting for each $E \subset X$

$$
\mu(E)=\sum_{k=1}^{\infty} \frac{1}{3^{k}} \nu\left(E X_{k}\right) ;
$$

obviously the transformation $\varphi$ of the space $X$ with the measure $\mu$ satisfies (7)-(9).

\section{References.}

[1] S. Banach, Théorie des opérations linéaires, Warszawa 1932.

2] Y.N. Dowker, Invariant measure and the ergodic theorems, Duke Mathematical Journal 14 (1947), p. 1051-1061.

[3] - A note on the ergodic theorem, Bulletin of the American Mathematical Society 55 (1949), p. 379-383.

[4] N. Dunford and D. S. Miller, On the ergodic theorem, Transactions of the American Mathematical Society 60 (1946), p. 538-549.

[5] S. Hartman, E. Marczewski et C. Ryll-Nardzewski, Théorèmes ergodiques et leurs applications, Colloquium Mathematicum 2 (1949-50), p. 109-123.

[6] F. Riesz, Sur la théorie ergodique, Commentarii Mathematici Helvetici 17 (1944-45), p. 221-239.

\section{PAŃSTWOWY INSTYTUT MATEMATYCZNY}

STATE INSTITUTE OF MATHEMATICS

(Reçu par la Rédaction le 17.11.1950). 